\title{
Tendencias temporales del cáncer de cuello uterino invasivo en mujeres entre 20 y 39 años en Manizales, Colombia. 2003-2018 ${ }^{\mathrm{i}}$
}

\author{
María del Pilar Arango Arango a \\ ${ }^{a}$ Médica. Ginecoobstetra, Clínica Santillana, Manizales. Docente, Universidad de Manizales. \\ ORCID: https://orcid.org/0000-0002-3119-283X, mariadelpilar.arango@yahoo.es \\ DOI: https://doi.org/10.22517/25395203.24621
}

\section{Resumen}

Objetivo: Analizar la tendencia de la incidencia de cáncer de cuello uterino invasivo en mujeres entre 20 y 39 años en Manizales, Colombia, 2003-2018.

Materiales y métodos: Estudio observacional, descriptivo, retrospectivo, de base poblacional. Se evaluaron las tendencias temporales en la incidencia (con referencia a Población Segi, por 100.000 mujeres/ año) utilizando la regresión de Joinpoint (Joint Point Regression Program v. 4.7.0.0 del National Cancer Institute de los EEUU) para estimar los cambios porcentuales anuales y años de variación significativa en las tendencias.

Resultados: Se realizó el estudio con un total de 116 pacientes, cuya edad promedio al momento del diagnóstico fue 33.7 años. Sólo se hallaron 3 casos de cáncer invasivo en mujeres entre 20 y 25 años. El mayor número de casos se presentó en el grupo de mujeres de 35 a 39 años. El tipo histológico más frecuente fue el carcinoma escamocelular. El $45.6 \%$ de los casos se diagnosticaron en etapas tempranas. El Cambio Porcentual Anual Promedio calculado mediante el modelo de regresión Joinpoint mostró un descenso de $7,9 \%$ en la tasa de incidencia entre 2003 y 2018, con un punto de quiebre en el año 2010. Conclusiones: El cáncer de cuello uterino en Manizales tiene escasa frecuencia en mujeres menores de 25 años y para edades entre 20 y 39 años muestra una tendencia a la disminución en el tiempo, en particular desde el año 2010 en adelante.

Palabras clave: Cáncer de cuello uterino, mujeres, adolescente, adulto joven, epidemiología, estudios epidemiológicos, tendencias (DeCS, BIREME)

\section{Introducción}

Según GLOBOCAN, en el año 2018, en el mundo se presentaron 570.000 nuevos casos y 311.000 muertes por cáncer de cuello uterino (1). En países de mayores ingresos, la mortalidad por cáncer de cuello uterino ha disminuido cerca de la mitad en los últimos 30 años, fenómeno atribuido a los programas de tamizaje. En los países de bajos recursos las tasas varían entre la estabilidad y el aumento (1,2). En América Latina, en el año 2015 , se reportaron 74.488 casos y 31.303 muertes, se estima un incremento de $45 \%$ en la mortalidad para el año 2030 (3).

Según la Agencia Internacional de Investigación en Cáncer (IARC) en Colombia para el año 2018 se presentaron 3.853 casos nuevos y 1.775 muertes (4). En el caso del departamento de Caldas, en el 2018 se reportaron 142 casos, de los cuales 98 fueron de Manizales (datos no publicados - Secretaría de Salud de Manizales). Los patrones de incidencia reflejan las tendencias en los comportamientos asociados con el riesgo de cáncer y los cambios en la práctica médica, como el uso de pruebas de detección del mismo (5).

Con el fin de reducir la incidencia de cáncer de cuello uterino invasivo se han desarrollado estrategias de prevención primaria mediante el uso de vacunas contra el VPH y de prevención secundaria mediante la detección temprana del riesgo debido a que la infección por ciertos tipos del Virus del Papiloma Humano (VPH) es una causa necesaria para el cáncer de cuello uterino $(2,3,6,7,8)$.

Entre 1990 y 2010, en el mundo se observó una disminución del $26,9 \%$ de la tasa de mortalidad por cáncer cervico-uterino y en los países desarrollados esta reducción ha sido sostenida desde 1.975 (9). En Colombia, las tasas estandarizadas por este tumor disminuyeron de 13,2 por 100.000 mujeres-año en la década de los 80's a 8,7 en el 2013 (10). 
En el año 2012 la Sociedad Americana de Patología Cervical y Colposcopia (ASCCP, por la sigla inglesa), publicó la nueva forma de cribado donde todas las mujeres deben comenzar las pruebas de detección a los 21 años. El grupo etario entre 21 - 29 años deben hacerse la prueba de citología cada 3 años y después de los 30 una citología combinada con una prueba de VPH cada 5 años. A esto se le llama prueba conjunta (o cotest) que se debe continuar hasta los 65 años $(7,11)$.

Para Colombia, la Guía de Práctica Clínica del Ministerio de Salud en el año 2014 estableció la realización de pruebas de ADN para la detección de VPH entre los 30 a los 65 años de edad. Además, recomienda el uso de citología para la clasificación diagnóstica (triage) de las pacientes positivas a las pruebas de ADN- VPH. Para las mujeres entre 25 y 30 años de edad, sugiere la tamización con citología cada 3 años ante resultados negativos. Teniendo en cuenta la baja incidencia de cáncer y lesiones precancerosas en mujeres menores de 25 años, los expertos sugieren no realizar tamización como parte de un programa poblacional organizado, dejando a criterio del clínico ofrecer un diagnóstico de oportunidad con citología a las mujeres de este grupo de edad (12).

De acuerdo con datos del Programa de Vigilancia, Epidemiología y Resultados Finales (SEER) del Instituto Nacional del Cáncer (NCI), Benard y cols. indican que de 1999 a 2008 un $21 \%$ de los cánceres invasivos en mujeres menores de 40 años correspondieron a mujeres entre 20 y 29 años y un $1 \%$ a mujeres menores de 20 años (13).

En el contexto latinoamericano, un estudio de base hospitalaria en Lima, Perú, encontró, entre las mujeres menores de 35 años, que el grupo etario más frecuente fue el de 30 a 35 años con $78,9 \%$ de cáncer de cuello uterino, seguido del grupo de 25 a 29 años con $16,0 \%$ y el de 20 a 24 años con 5,3\% (14).

Dado que la estrategia de tamización en Colombia puede dejar descubierta a la población femenina entre los 15 a 25 años, en quienes, de acuerdo con las recomendaciones actuales, no se debería aplicar ninguna prueba de tamización, el objetivo de este estudio es analizar las tendencias temporales del cáncer de cuello uterino invasivo en mujeres de 20 a 39 años durante el período 2003 - 2018 en Manizales, Colombia.

\section{Materiales y métodos}

Estudio observacional descriptivo, de cohorte de casos incidentes, retrospectivo, que incluyó 119 casos de cáncer invasivo de cuello uterino en mujeres entre 20 y 39 años, identificados por el Registro Poblacional de Cáncer de Manizales.

Se consultó base de datos maestra y se utilizaron filtros por localización C53 y edad para menores de 40 años. Se verificó la fecha de nacimiento de las pacientes y se corrigió la edad al momento del diagnóstico. Del total de casos se excluyeron 2 que no tenían cáncer invasivo y 1 que superaba el margen de edad. Los datos del registro poblacional se verificaron con reportes médicos y fuentes de información gubernamentales, además, se completaron datos para construir el estadio clínico al momento del diagnóstico con base en la clasificación de la Federación Internacional de Ginecología y Obstetricia (FIGO) 2018.

Se retroalimentó la información sobre el lugar de residencia al momento del diagnóstico (dirección o barrio) a partir de la cual se asignó el nivel socio económico con base en el estrato predominante del barrio de residencia según la base de datos ViviendasHogares-Personas (VIHOPE) del Departamento Administrativo Nacional de Estadística - DANE. Se completó y validó el estadio clínico y los diagnósticos histológicos con base en la Clasificación Internacional de Enfermedades para Oncología, primera revisión de la tercera edición (CIE-O 3.1).

Se realizó descripción univariada de las variables de interés, además de análisis bivariado para las variables de estadio clínico con edad y estrato socioeconómico. La variable estadio clínico fue dicotomizada como estadio temprano para los casos con estadios I y II de la FIGO y como estadio tardío para los demás. Como punto de corte para la edad se utilizó la mediana de la edad de todo el conjunto de datos, es decir, menores de 34 años y mayores de 34 . La variable nivel socio-económico se categorizó como variable ordinal en tres grupos: alto (estratos 5 y 6), medio (estratos 3 y 4 ) y bajo (estratos 1 y 2).

Para el ajuste de tasas por edad se utilizó el método de estandarización directo; se establecieron cuatro estratos seleccionados para fines de esta investigación (grupos de edad quinquenales entre 20 y 39 años) y se utilizó como referencia la población mundial de Segi. 
Los datos de población en riesgo se obtuvieron de las proyecciones poblacionales oficiales del Departamento Administrativo Nacional de Estadística.

Las tendencias temporales en la incidencia se estimaron utilizando la regresión de Joinpoint, la cual emplea regresión loglineal por partes para evaluar los cambios porcentuales anuales e identificar cualquier año en el que ocurra un cambio significativo en las tendencias, se utilizó la versión 4.7.0.0 del Joinpoint Regression Program producido por el Instituto Nacional de Cáncer (NCI, USA).

Finalmente, para el procesamiento y análisis de los datos fueron utilizados los paquetes Microsoft Excel@; SPSS $®$ versión 22 (licenciado para la Universidad de Caldas) y Stata IC 14.2 (licencia personal). A nivel ético, esta investigación contó con la aprobación del Comité de Bioética de la Facultad de Ciencias para la Salud de la Universidad de Caldas.

\section{Resultados}

La mediana de la edad al momento del diagnóstico fue de 34.2 años (rango intercuartílico - RIC=6.6años). Se encontró que la mayoría de las pacientes de este estudio pertenecen a nivel socioeconómico bajo y medio en el $38,8 \%$ y $39,7 \%$, respectivamente. Solo el $4,3 \%$ de las mujeres pertenecen al estrato socioeconómico alto. Para esta variable, el porcentaje de casos con dato perdido alcanzó el $17,2 \%$. Con relación a la distribución por grupos de edad, se evidencia que los casos de cáncer invasivo se concentran en el grupo de 35 a 39 años seguido del grupo de 30 a 34 años. Una minoría de los casos se presentan en mujeres menores de 30 años, y solo 3 casos ocurrieron en menores de 25 años. (ver figura 1)
EDAD AL DIAGNÓSTICO

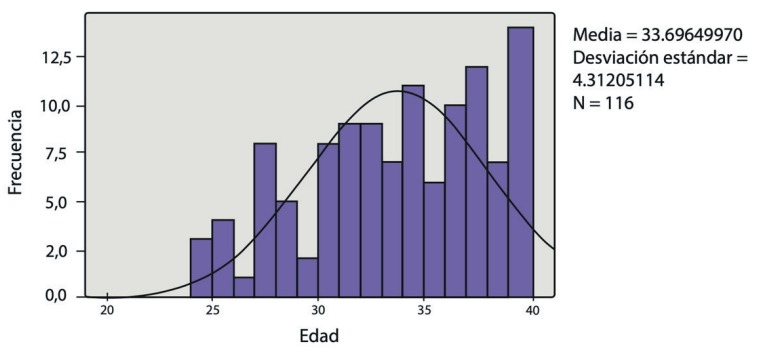

Figura 1. Distribución por edad de las pacientes con cáncer de cuello uterino entre 20 y 39 años. Manizales, Colombia, 2003-2018

Fuente : Elaboración propia.

El número absoluto de casos por año varió entre los períodos 2003 - 2010 y 2011- 2018, observándose una disminución en el último período. Los años con mayor número de casos incidentes fueron 2005 y 2008-2009, mientras que el menor número de casos se observó en el año 2015.

De acuerdo con la clasificación morfológica, el tipo histológico más frecuente fue el carcinoma escamocelular, seguido del adenocarcinoma. Más del $10 \%$ de los casos no tuvieron una clasificación morfológica adecuada.

En cuanto a la estadificación clínica al diagnóstico, el $45,6 \%$ de las pacientes se clasificaron en estadio temprano y el $24,1 \%$ en estadio avanzado. La distribución de casos incidentes por estadio diagnóstico fue de $33(28,4 \%), 20(17,2 \%), 21(18,1 \%)$, y $7(6,0 \%)$ para los estadios I al IV, respectivamente; pero hubo un volumen de 35 casos $(30,2 \%)$ en los cuales este dato no se pudo encontrar.

Con respecto a la relación entre la etapa al momento del diagnóstico y el nivel socioeconómico, se observó una mayor proporción de mujeres con estadios tempranos en el grupo de nivel socioeconómico alto y una mayor proporción de estadios tardíos en el de nivel bajo (Prueba exacta de Fisher, valor $\mathrm{p}=0,034$ ). Se exploró también la asociación entre estadificación clínica (temprana o tardía) y los grupos de edad, sin encontrar asociación estadísticamente significativa (Prueba exacta de Fisher, valor $\mathrm{p}=0,89$ ). 
TASA AJUSTADA POR EDAD SEGÚN TIPO HISTOLÓGICO

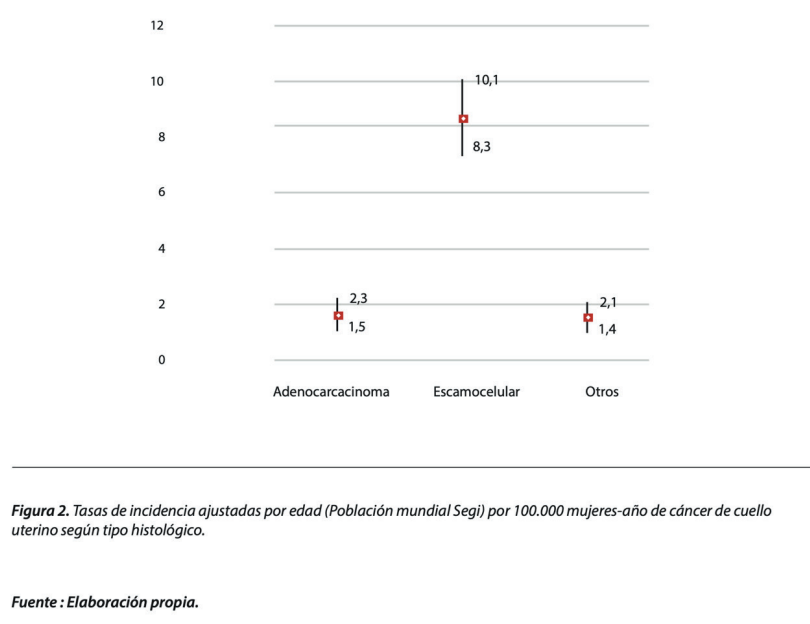

En la figura 2 se muestran las tasas de incidencia de cáncer de cuello uterino ajustadas por edad (población mundial Segi) por 100.000 mujeres/año según tipo histológicoi. Se destacan las tasas específicas por grupos de edad, cuya distribución en todos los tipos histológicos agregados fue: 1,2 entre 20 a 24 años; 7,2 entre 25 a 29 años; 18,6 entre 30 a 34 años; y 22,7 entre 35 a 39 años.

En cuanto al comportamiento de la incidencia por grupos de edad, en el carcinoma escamocelular, la tasa fue de 0,4 por 100.000 entre 20 y 24 años; de 6,1 en el grupo de 25 a 29 años; 14,3 entre 30 y 34 años; y de 15,9 entre 35 y 39 años. Para adenocarcinoma, las tasas específicas en estos mismos grupos de edad fueron 0,4 ; 0,$0 ; 3,0 ;$ y 3,6 respectivamente. En lo referente a los otros tipos histológicos, las tasas fueron muy similares a las de adenocarcinoma.

En la figura 3 y 4 se muestra un descenso del 7,97\% anual en la tasa de incidencia ajustada por edad en las mujeres con cáncer de cuello uterino invasivo entre 20 a 39 años. Modelos alternativos mostraron 2 puntos de quiebre que no fueron significativos. El descenso significativo en la tasa se observa más claramente a partir de 2010.

i. Datos de incidencia del año 2018 son preliminares por lo que la incidencia final estimada para este año puede ser mayor en el momento cuando se complete la recolección de los datos.

\section{CAMBIO PORCENTUAL ANUAL}

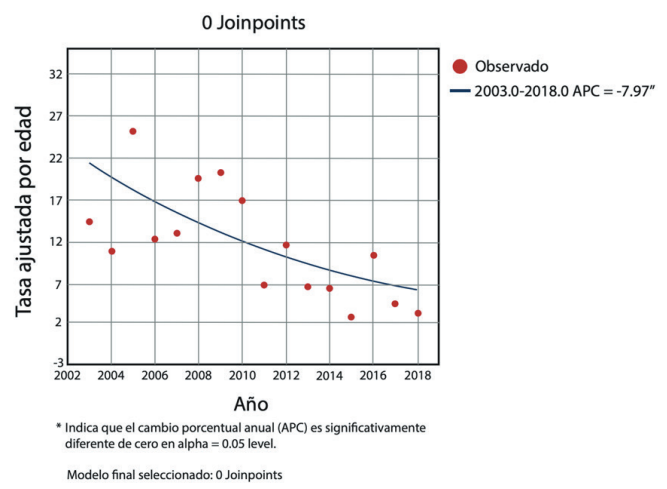

Figura 3. 0 Joint point . Muestra un descenso 7,97 porcentual anual en la tasa de incidencia ajustada por edad en las mujeres con cáncer de cuello uterino entre 20 y 39 años.

Fuente : Elaboración propia.

\section{TENDENCIAS TEMPORALES}

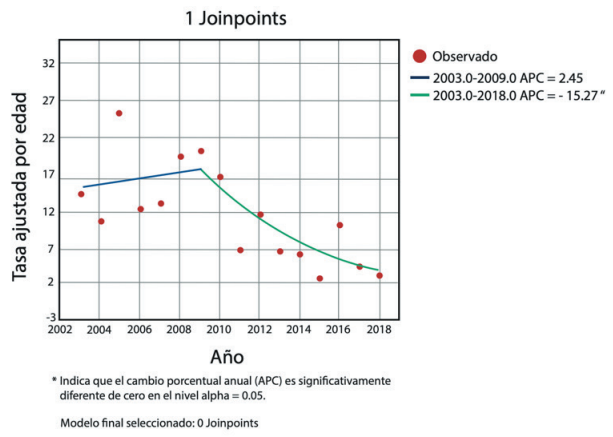
Figura 4 Joint point puntos de quiebre del valor porcentual anual en la tasa de incidencia ajustada por edad en las
mujeres con cáncer de cuello uterino entre 20 y 39 años.

Fuente: Elaboración propic

\section{Discusión}

Los resultados de esta investigación, en términos de la distribución de casos por grupos de edad, son similares a los encontrados en Estados Unidos entre 1999 y 2008, donde el $78 \%$ de los casos de cáncer de cérvix se diagnosticaron en mujeres con edades de 30 a 39; el $21 \%$ se diagnosticó en mujeres de 20 a 29 años y el $1 \%$ en menores de 20 años. Hasta los 14 años las tasas de incidencia fueron cercanas a 0 y se eliminaron de las tablas debido a los bajos recuentos de casos (13). 
En este estudio la concentración de casos en el grupo de edad de 30 a 39 años refuerza la necesidad de implementar lo antes posible la tamización por detección con citología en las jóvenes mayores de 25 años y tipificación de VPH en las mayores de 30 años, utilizando además modelos de atención que permitan adecuadas coberturas de la población en riesgo. Los programas organizados de detección para el cáncer de cuello uterino son herramientas eficaces y poderosas que pueden prevenir muertes como se ha demostrado en Europa, América del Norte y Asia (15).

Una investigación de carácter descriptiva del comportamiento del cáncer de cuello uterino en jóvenes menores o iguales a 35 años entre 1990 y 2012 en EEUU, de 126 pacientes con las que se realizó el estudio, el $15 \%$ estaban en edades menores o iguales a 25 años y el $85 \%$ tenían edades comprendidas desde 26 hasta 35 años (16). En este estudio, fue menor el porcentaje de casos en las más jóvenes, de 116 pacientes el $3 \%$ de los casos se presentaron en mujeres menores de 25 años y el $97 \%$ de los casos en mayores de 25 años.

Varios estudios epidemiológicos muestran que la incidencia de cáncer de cuello uterino en mujeres jóvenes tiende al aumento $(16,19,20,21,22)$. Un estudio realizado en el Reino Unido demostró que la incidencia del cáncer de cuello uterino en las mujeres jóvenes de 20 a 29 años aumentó anualmente 10,3\% entre los años 2000 y 2009 (17). De igual forma, en Japón, entre los años 1985 y 2012, se evidencia un aumento en las tasas de incidencia para cáncer de cuello uterino en mujeres de 25 a 49 años. Igualmente, en Corea del Sur en el mismo período aumentó la tasa de incidencia en las jóvenes entre 20 y 29 años (18).

En Costa Rica, encontraron aumento de la incidencia de cáncer de cuello uterino desde el 2012. El grupo de edad más afectado de manera global (ca in situ y ca invasor) fue el de 26 a 30 años; pero para cáncer invasor fue el grupo de 46 a 50 años (23). Es necesario considerar que, al incluir carcinomas in situ, la incidencia en dicho estudio podría haber aumentado producto de un buen funcionamiento de los programas de tamización y no por un aumento real de la incidencia.

Un estudio en un hospital londinense reportó que de 108 casos de cáncer invasivo diagnosticados entre 1999 y 2006, 2 casos ocurrieron en mujeres de 20-24 años, 12 entre 25 y 29 años y 18 entre 30 y 34 años; el $62.1 \%$ fueron detectados en estadio I según clasificación
FIGO (24). Estos resultados también son consistentes con los hallados para esta investigación.

Se considera que el cribado del cáncer de cuello uterino en mujeres menores de 21 años es menos efectivo que en los grupos de mayor edad, por lo que se ha aumentado la edad para comenzar la evaluación, independientemente del inicio de la actividad sexual. Un estudio estadounidense comenta que, aunque la detección ocurre con menos frecuencia en grupos de edad más joven (15-19 años) se realizaban 2.7 millones de pruebas de citología para detectar un promedio de 14 cánceres que ocurrían anualmente (13).

El bajo nivel socioeconómico se asocia con un mayor riesgo de cáncer de cuello uterino. De 1988 a 1992 en los Estados Unidos, la incidencia de cáncer de cuello uterino fue mayor en las mujeres que vivían en comunidades con niveles de pobreza más altos $(\geq 20 \%$ o más de la población por debajo del nivel de pobreza: 19.2 casos por 100,000 mujeres versus $<10 \%$ por debajo del nivel de pobreza: 8,8 por 100.000). Las mujeres en los condados de alta pobreza en comparación con los de baja pobreza tenían una tasa de mortalidad por cáncer de cuello uterino $71 \%$ más alta (25). Lo anterior es similar a lo hallado en este estudio, donde más de dos terceras partes de las pacientes incluidas en el estudio pertenecen a estratos medio y bajo.

En referencia particular al adenocarcinoma invasivo y sus variantes histológicas, su incidencia ha aumentado en las últimas décadas, en especial en mujeres más jóvenes. De hecho, en los últimos 35 años, ha habido un aumento del 32 por ciento de adenocarcinoma y un aumento del 16 por ciento en las histologías adenoescamosas entre las pacientes con cáncer de cuello uterino recién diagnosticadas (25). En contraste, en esta investigación se encontraron datos diferentes en las mujeres entre 20 y 30 años, para estas jóvenes el tipo histológico predominante fue el carcinoma escamocelular y no el adenocarcinoma u otros tipos histológicos.

La incidencia del cáncer de cuello uterino, incluyendo carcinoma de células escamosas, ha venido disminuyendo en los países desarrollados. Sin embargo, el adenocarcinoma se ha incrementado entre jóvenes como lo demuestran Birgitte y Col en el estudio realizado entre 1997 y 2011 en Dinamarca (26).

En Corea del Sur, se ha reportado un cambio porcentual anual de 3,9\% (IC 95\%: $-4,2$ a $-3,6$ ) 
entre 1993 y 2012, además de la presencia de efecto cohorte y de un leve aumento en la tasa de incidencia en las mujeres menores de 30 años (14). Contrastando los datos, para Manizales, la reducción del cambio porcentual anual fue casi el doble que en de Corea, lo que se puede explicar, primero, porque en el año 2018 no se ha cerrado la recolección de datos del Registro Poblacional, y puede no haberse completado la exhaustividad del registro poblacional, con lo cual la tasa del último año puede ser falsamente baja, segundo, porque Manizales contaba con tasas más altas que Corea al inicio del registro y por lo tanto es más visible el cambio que en Corea que partía de tasas más bajas en 1993 y, tercero, porque el departamento de Caldas ha tenido desde el año 2000 un programa descentralizado de tamización con Colposcopia en atención primaria, con 17 muertes anuales por 100.000 mujeres año menos al ser comparado con el programa centralizado de atención (27), lo que pudo estar relacionado con el notorio descenso en la incidencia del Cáncer invasivo en Manizales.

\section{Conclusiones}

Dado que en esta investigación se utilizaron datos históricos de los años 2003 a 2018 del registro poblacional de cáncer de Manizales, se recomienda para otros trabajos, continuar estudiando estas variables con la información dispuesta en el registro municipal desde el año 2016 que cuenta con la notificación obligatoria de los casos de cáncer de cuello uterino invasivo en Colombia y, por lo tanto, podría complementar los datos capturados por el Registro Poblacional.

Se concluye que el cáncer de cuello uterino en Manizales tiene escasa frecuencia en mujeres menores de 25 años y para edades entre 20 y 39 años muestra una tendencia a la disminución en el tiempo, en particular desde el año 2010. Se encuentra un mayor número de casos de cáncer del tipo escamocelular en mujeres jóvenes. Hay mayor número de cánceres invasivos en el grupo de mujeres de 30-39 años por lo que se deben reforzar intervenciones para la prevención del cáncer.

En las últimas décadas las tasas de incidencia por cáncer de cuello uterino han disminuido en Manizales probablemente debido a los programas descentralizados de detección basados en la población. Se requieren nuevas políticas públicas sumadas a la investigación que permitan retroalimentar al proveedor de la salud para poder brindar atención segura y de calidad a las pacientes jóvenes.

\section{Agradecimientos}

Al doctor Nelson Enrique Arias Ortiz, por su dirección durante el proceso de investigación, a la Universidad de Caldas y al Registro Poblacional de Cáncer de Manizales que facilitaron la información.

\section{Referencias}

1. Bray F, Ferlay J, Soerjomataram I, Siegel RL, Torre LA, Jemal A. Global cancer statistics 2018: GLOBOCAN estimates of incidence and mortality worldwide for 36 cancers in 185 countries. CA Cancer J Clin. 2018;68(6):394-424.

2. Cohen PA, Jhingran A, Oaknin A, Denny L. Cervical cancer. 2019;393(10167):169-82.

3. Bychkovsky BL, Ferreyra ME, Strasser-Weippl K, Herold CI, de Lima Lopes GJr,Dizon DS, Schmeler KM, Del Carmen M, Randall TC, Nogueira-Rodrigues A, de Carvalho Calabrich AF, St Louis J, Vail CM, Goss PE. Cervical cancercontrol in Latin America:Acall to action. Cancer. 2016;122(4):502-14. doi: 10.1002/cncr.29813.

4. International Agency for Research on Cancer. Colombia. Source: Globocan. [internet]2020 [citado el 15 de marzo 2020]. Disponible en: $\quad$ https://gco.iarc.fr/today/data/factsheets/ populations/170-colombia-fact-sheets.pdf

5. Siegel RL, Miller KD, Jemal A. Cancer statistics. Cancer J Clin. 2019;69(1):7-34.

6. Muñoz N, Bravo LE. Epidemiology of cervical cancer in Colombia. Salud Publica Mex. 2014;56(5):431-9.

7. Tota JE, Bentley J, Blake J, Coutlée F, Duggan MA, Ferenczy A, et al. Introduction of molecular HPV testing as the primary technology in cervical cancer screening: Acting on evidence to change the current paradigm. Prev Med.2017;98:5-14.

8. Vidal C, Hoffmeister L, Biagini L. Tendencia de la mortalidad por cáncer de cuello uterino en Chile: aplicación de modelos de regresión joinpoint. Rev panam salud pública. 2013;33(6):407-13. 
9. Pardo C, de Vries E. Breast and cervical cancer survival at instituto nacional de cancerología, Colombia. Colomb Med. 2018;49(1):102-8.

10. Saslow D, Solomon D, Lawson HW, Killackey M, Kulasingam SL, Cain J, et al. American Cancer Society, American Society for Colposcopy and Cervical Pathology, and American Society for Clinical Pathology screening guidelines for the prevention and early detection of cervical cancer. CA Cancer J Clin. 2012;62(3):147-72. doi: 10.3322/caac.21139.

11. Practice Bulletin No. 157: Cervical Cancer Screening and Prevention. Obstet Gynecol. 2016;127(1):e120. doi: 10.1097/AOG.0000000000001263.

12. Ministerio de Salud y Protección Social. Guía de práctica clínica (GPC) para la detección y manejo de lesiones precancerosas de cuello uterino. Guía completa. Colombia [internet] 2014 [citado el 15 de marzo 2020]. Disponible en: https://www.cancer.gov. co/Gu\%C3\%ADas-y-Protocolos/Gu\%C3\%ADasde-Practica-clinica/LPC-Guia-profesionales.pdf

13. Benard VB, Watson M, Castle PE, Saraiya M. Cervical carcinoma rates among young females in the United States. Obstet Gynecol. 2012;120(5):1117-23.

14. Ruiz R, Serrano M, Ruiz EF, Mantilla R, Valdivieso N, Olivera M, et al. Clinical-pathological features and survival in young women with cervical cancer: A retrospective analysis from the instituto nacional de enfermedades neoplÁsicas. Rev Peru Med Exp Salud Publica. 2017;34(2):218-27.

15. Moon EK, Oh CM, Won YJ, Lee JK, Jung KW, Cho $\mathrm{H}$, et al. Trends and age-period-cohort effects on the incidence and mortality rate of cervical cancer in Korea. Cancer Res Treat. 2017;49(2):526-33.

16. Pelkofski E, Stine J, Wages NA, Gehrig PA, Kim KH, Cantrell LA. Cervical Cancer in Women Aged 35 Years and Younger. Clin Ther. 2016;38(3):459-66.

17. Patel A, Galaal K, Burnley C, Faulkner K, Martin-Hirsch P, Bland MJ, et al. Cervical cancer incidence in young women: A historical and geographic controlled UK regional population study. $\quad \mathrm{Br} \quad \mathrm{J}$ Cancer. 2012;106(11):1753-9.

18. Utada M, Chernyavskiy P, Lee WJ, Franceschi S,
Sauvaget $\mathrm{C}$, de Gonzalez $\mathrm{AB}$, et al. Increasing risk of uterine cervical cancer among young Japanese women: Comparison of incidence trends in Japan, South Korea and Japanese-Americans between 1985 and 2012. Int J Cancer. 2019;144(9):2144-52.

19. Kong Y, Zong L, Yang J, Wu M, Xiang Y. Cervical cancer in women aged 25 years or younger: A retrospectivestudy.CancerManagRes.2019;11:2051-8.

20. Berkowitz RS, Ehrmann RL, Lavizzo-Mourey R, Knapp RC. Invasive cervical carcinoma in young women. Gynecol Oncol. 1979;8(3):311-6.

21. Elliott PM, Tattersall MH, Coppleson M, Russell P, Wong F, Coates AS, et al. Changing character of cervical cancer in young women. BMJ. 1989;298(6669):288-90.

22. Sasieni P, Castanon A. Dramatic increase in cervical cancer registrations in young women in 2009 in England unlikely to be due to the new policy not to screen women aged 20-24. J Med Screen. 2012;19(3):127-32.

23. Caro-Porras N. Análisis epidemiológico de mujeres con carcinoma epidermoide de cérvix, 2007-2015. Acta méd costarric. 2017;59(1):22-7.

24. Herbert A, Holdsworth G, Kubba AA. Cervical screening: Whyyoungwomenshouldbeencouraged tobe screened.JFamPlanReprodHealCare.2008;34(1):21-5.

25. Frumovitz MD. Invasive cervical cancer: Epidemiology, risk factors, clinical manifestations, and diagnosis. Post TW, ed. UpToDate. Waltham, MA: UpToDate Inc. 2020.

26. Baldur-Felskov B, Munk C, Nielsen TSS, Dehlendorff C, Kirschner B, Junge J, et al. Trends in the incidence of cervical cancer and severe precancerous lesions in Denmark, 1997-2012. Cancer Causes Control. 2015;26(8):1105-16.

27. Ocampo Ramírez, J. Análisis costo efectividad de estrategias de atención para cáncer de cuello uterino: un estudio de caso para el departamento de Caldas. [Internet]. Montevideo, Uruguay: Universidad de la República ; 2018-07-05. [citado diciembre 2020]. Universidad Nacional de Colombia, Sede Medellín :Facultad de Ciencias Humanas y Económicas, Escuela de Economía. 